\title{
Lumen
}

Selected Proceedings from the Canadian Society for Eighteenth-Century Studies

\section{Germaine de Staël, citoyenne du monde : le cosmopolitisme dans l'oeuvre staëlien}

\section{Laetitia Saintes}

Volume 38, 2019

URI : https://id.erudit.org/iderudit/1059273ar

DOI : https://doi.org/10.7202/1059273ar

Aller au sommaire du numéro

Éditeur(s)

Canadian Society for Eighteenth-Century Studies / Société canadienne d'étude du dix-huitième siècle

ISSN

1209-3696 (imprimé)

1927-8284 (numérique)

Découvrir la revue

Citer cet article

Saintes, L. (2019). Germaine de Staël, citoyenne du monde : le cosmopolitisme dans l'oeuvre staëlien. Lumen, 38, 73-88. https://doi.org/10.7202/1059273ar 


\title{
Germaine de Staël, citoyenne du monde : le cosmopolitisme dans l'œuvre staëlien
}

\author{
LAETitia SAINTES \\ Université catholique de Louvain
}

\begin{abstract}
Enfin il reste encore une chose vraiment belle et morale, dont l'ignorance et la frivolité ne peuvent jouir: c'est l'association de tous les hommes qui pensent d'un bout de l'Europe à l'autre. Souvent ils n'ont entre eux aucune relation; ils sont dispersés, souvent à grandes distances l'un de l'autre; mais quand ils se rencontrent un mot suffit pour qu'ils se reconnaissent. Ce n'est pas telle religion, telle opinion, tel genre d'étude, c'est le culte de la vérité qui les réunit ${ }^{1} \ldots$
\end{abstract}

Germaine de Staël, De l'Allemagne.

\section{Coppet, creuset libéral et cosmopolite du romantisme}

De tous les groupes littéraires qui ont marqué l'histoire des lettres, le Groupe de Coppet, constitué autour de Germaine de Staël, est sans conteste l'un des plus atypiques. Loin de former une société codifiée pourvue d'objectifs définis, de statuts, comme une académie, d'une revue, ou même d'une doctrine donnée, le Groupe ne constitue ni un parti politique, même s'il se passionne pour ce sujet, ni une secte, bien que la religion intéresse certains de ses membres. Fort de sa diversité, il ne se prononce pas pour une forme littéraire précise, comme plus tard les naturalistes ${ }^{2}$, et est loin de se cantonner à un seul domaine:

1. Germaine de Staël, De l'Allemagne, établissement du texte, notes et avantpropos d'Axel Blaeschke, Paris, Garnier, coll. «Classiques Jaunes », 2014, t. II, p. 229230.

2. Simone Balayé, «Le Groupe de Coppet: conscience d'une mission commune ", dans Simone Balayé et Jean-Daniel Candaux (dir.), Le Groupe de Coppet. Actes et documents du deuxième Colloque de Coppet. 10-13 juillet 1974, Genève/Paris, Slatkine/Champion, 1977 , p. 29-45; ici p. 30. 
philosophie, histoire, économie, littérature se côtoient et s'entremêlent dans un projet (et un propos) résolument pluridisciplinaire. Son noyau dur rassemble ainsi des écrivains dont certains joueront un rôle politique actif (Benjamin Constant, Prosper de Barante, Elzéar de Sabran), des économistes (Jean de Sismondi), des philosophes (Bonstetten, Schlegel), des femmes de lettres (Albertine Necker de Saussure, Juliette Récamier), en plus des enfants de Germaine de Staël, très tôt amenés à participer à la discussion.

Les rapports de ses membres se fondent non sur une doctrine commune, mais sur une convergence à la fois philosophique, morale et politique 3 . Lappellation de «Groupe de Coppet» a d'ailleurs été forgée a posteriori : ses membres ne se sont jamais de leur vivant organisés en un groupe formellement défini. Doté d'un prestige intellectuel inégalé - on connaît le mot de Bonstetten à ce propos, selon lequel «[i]l se dépense plus d'esprit à Coppet en un jour que dans maints pays en un $\mathrm{an}^{4} »-$, le Groupe diffère enfin des salons du XviII siècle, bien qu'il en procède en partie, comme on le verra, et des groupes littéraires nationaux (comme ceux d'Iéna ou de Weimar) ${ }^{5}$, puisqu'il se caractérise avant tout par son caractère cosmopolite.

En dépit de l'hétérogénéité de ses membres et de leurs domaines d'élection, le Groupe trouve sa cohésion dans la personne de Germaine de Staël, inspiratrice et point de ralliement. Seule femme dans l'histoire littéraire à avoir joué un tel rôle tout en poursuivant ses travaux ${ }^{6}$, celle-ci prône non l'orthodoxie, mais la liberté de penser et la diversité, mot d'ordre du Groupe. À cet égard, la conversation, lieu forcément dialogique, donc potentiellement subversif, figure sans conteste l'activité la plus caractéristique de l'esprit du Groupe.

Le Groupe prend racine dès les débuts de Germaine de Staël comme salonnière, en 1786. Réunissant écrivains, aristocrates, diplomates, hauts fonctionnaires, l'écrivaine traite avant tout de politique

3. Roland Mortier, «Les États Généraux de l'opinion européenne », dans Kurt Kloocke (dir.), Le Groupe de Coppet et l'Europe. Actes du cinquième Colloque de Coppet, 8-1o juillet 1993, publ. avec la collab. de Simone Balayé, Lausanne/Paris, Institut Benjamin Constant/Jean Touzot, 1994, p. 17-24; ici p. 19.

4. «Lettre de Charles Victor de Bonstetten à Friederike Brun, août 1804", dans Briefe von Karl Viktor von Bonstetten an Friederike Brun, établissement du texte par Friedrich von Matthisson, Francfort-sur-le-Main, W. Schaefer, 1829, p. 213.

5. Simone Balayé, «Le Groupe de Coppet», art. cit., p. 30.

6. Id. 
jusqu'en 1792. C'est à la suite de son premier exil, dû aux troubles révolutionnaires, que ce qui était jusqu'alors un salon parisien, certes brillant mais similaire par sa forme à d'autres cercles intellectuels du temps, entame sa métamorphose. À compter de cette date, il ne se tiendra plus à Paris que de façon épisodique, et choisit Coppet - «centre de lumière, de religion, de fortune» selon le mot de Barante - comme lieu d'élection, et Genève comme nouveau centre. De façon apparemment paradoxale, c'est cet exil et ce confinement forcés dans une ville au carrefour de l'Europe, accessible et visitée de toutes parts, qui donneront au cercle de Germaine de Staël une dimension et une renommée internationales ${ }^{7}$ - comme si la sociabilité, se voyant interdire le séjour parisien, devait dès lors prendre place à l'échelle européenne. D'emblée, donc, le Groupe incarne «l'esprit européen »; Stendhal l'a bien compris, qui, peu après le décès de Germaine de Staël, décrira les réunions de Coppet comme les «états généraux de l'opinion européenne ${ }^{8}$ ».

Les arrivées successives de Benjamin Constant (1794), de Wilhelm von Humboldt (1798), de Sismondi (1800) et d'Auguste Schlegel (1804), à la suite du séjour de Germaine de Staël dans le cercle de Weimar, mènent le Groupe vers de nouvelles orientations littéraires, accélérant par-là la métamorphose qui l'amènera à incarner l'essence même de l'esprit cosmopolite. Ainsi, les années 1805-1810, celles de l'apogée, voient naître les œuvres les plus abouties du Groupe, qui inaugurent en France une littérature nouvelle en contribuant très largement au premier romantisme français: Corinne (1807), les Recherches sur la nature et les lois de l'imagination (1807) de Bonstetten, le Wallstein (1809) de Constant ou encore De l'Allemagne (1810) paraissent alors, qui donnent une idée de la variété des sujets brassés et de l'importance de la recherche littéraire menée par le Groupe de Coppet.

Largement ouvert, le Groupe compte des membres de nationalités ${ }^{9}$ diverses, issus de la plupart des pays d'Europe; surtout, il déploie un cosmopolitisme unique en son genre: chacun des membres du Groupe étant enraciné dans un terroir, il s’agit pour eux de préserver les particularismes nationaux, d'utiliser les richesses propres à leur nation pour

7. Ibid., p. 31.

8. Stendhal, Rome, Naples et Florence, Paris, Julliard, 1964, p. 208-209.

9. Terme d'ailleurs inventé à Coppet. 
l'enrichissement de tous. Dans cette optique, on ne cherchera donc pas à gommer les différences en faveur d'une uniformité quelconque; au contraire, il s'agira de les exalter dans une visée commune d'enrichissement mutuel, de perfectibilité. De cette façon, comme l'a justement relevé Simone Balayé, le Groupe offre une combinaison inédite du culte de l'universel, propre au XVIII ${ }^{\mathrm{e}}$ siècle, et de celui de la différence, notion profondément romantique ${ }^{10}$.

Cette célébration des particularismes est également une invitation à sonder les réalités nationales autres. Dans cette perspective, nombre de membres du Groupe, dont Germaine de Staël, voyagent pour se doter de nouvelles connaissances, concrètes celles-ci, sur la mentalité des autres peuples, leur littérature, leurs idées philosophiques et politiques; aussi la division Nord/Midi reprise dès 1800 par l'inspiratrice du Groupe - et dans laquelle la géographie intellectuelle de l'Europe prend tout son sens ${ }^{11}$ - repose-t-elle sur une véritable expérience du terrain. Au cours de leurs voyages, les membres du Groupe en viennent en effet à concevoir les nations du Nord comme celles de la réflexion, de la lucidité et de la critique, celles où l'intelligence a le plus de force; Bonstetten, Staël et Sismondi forgent sur cette base un «mythe du Nord» qui doit beaucoup à celui façonné par les Lumières et s'inscrit dans la lignée de la pensée de Montesquieu ${ }^{12}$. C'est par cette expérience, par ces observations que le Groupe de Coppet forge sa conscience de la pluralité des nations, mais également de l'originalité de chaque culture et de la nécessité que ces cultures se soutiennent mutuellement pour évoluer ${ }^{13}$. Le cosmopolitisme de Coppet relève donc à la fois de l'idéal et du phénomène concret, du modèle théorique et de la réalité empirique, en ce qu'il consiste en la mobilisation réelle, à visée universelle, d'une connaissance des singularités nationales basée sur l'expérience.

Le Groupe est également à la charnière de deux siècles par son mode de communication; Coppet fait en effet de la conversation, mot-clé du XVIII siècle, le lieu central de l'échange des idées: on écrit,

10. Simone Balayé, «Le Groupe de Coppet», art. cit., p. 32.

11. Voir Jean Roussel, «L'ambiguïté des Lumières à Coppet», dans Simone Balayé et Jean-Daniel Candaux (dir.), Le Groupe de Coppet, op. cit., p. 171-184; ici p. 175 .

12. Voir ibid., p. 176.

13. Voir Simone Balayé, «Le Groupe de Coppet», art. cit., p. 32-33. 
on discute de ses ouvrages en cours - dans le but, toujours, de les améliorer, de les enrichir par des idées différentes des siennes, avec pour objectif que l'échange profite au plus grand nombre. Certains ouvrages, comme De la littérature (1800), Wallstein et De l'Allemagne, ont été composés au départ des conversations du Groupe ${ }^{14}$, d'où une création pleinement originale et plurielle. Toutefois, si la conversation enrichit nettement les œuvres des membres du Groupe, elle ne les amène pas à transformer leurs textes en profondeur et à altérer par-là ce qui fait leur spécificité - de la même façon que le cosmopolitisme du Groupe préserve l'intégrité des patries et des peuples dans le sens d'un enrichissement mutuel, et non d'une uniformisation qui annihilerait leurs différences et particularités ${ }^{15}$.

Il faut également citer parmi les modalités de ce cosmopolitisme unique l'accent mis sur la circulation des savoirs, au cœur du projet de Coppet. Cela passe par la correspondance et l'échange de livres (de l'école romantique surtout) et de revues par-delà les frontières et la censure, qui contribuent à créer un réseau intellectuel d'envergure internationale; au plus fort des persécutions impériales, Germaine de Staël évoque d'ailleurs l'exil comme un «tombeau seulement où la poste arrive ${ }^{16} »$. Cette circulation des savoirs, résolument cosmopolite, passe aussi par un usage unique de la traduction, dont le Groupe fait à la fois « un instrument essentiel de communication entre les cultures et les peuples et un objet de réflexion ${ }^{17}$ ». Sans le Groupe de Coppet, l'Allemagne romantique, mais aussi Dante, n'auraient en effet pas été accessibles aux Français aussi rapidement, et la génération de 1830 n'aurait pas été celle que l'on connaît ${ }^{18}$. Il faut à nouveau noter à cet égard l'importance de la mise en contact du Groupe avec la culture allemande, qui valorise une traduction fidèle des œuvres étrangères et ne les juge pas via une grille d'analyse classique; le Groupe traduit ainsi, comme le pointe Stephen Bann, pour «faire passer du nouveau,

14. Voir ibid., p. 33.

15. Voir id.

16. «Lettre à Madame Récamier, 6 janvier 1811 ", dans Germaine de Staël, Correspondance générale. "La destruction de De l'Allemagne. L'Exil à Coppet» (9 mai 1809-23 mai 1812), établissement du texte, notes et avant-propos de Béatrice W. Jasinski et Othenin d'Haussonville, Paris/Genève, Champion/Slatkine, 2oo8, t. VII, p. 353 .

17. Simone Balayé, «Le Groupe de Coppet», art. cit., p. 34.

18. Voir id. 
non pour faire figurer autrui à l'image du classicisme français ${ }^{19}{ }^{\text {». La }}$ traduction telle que le Groupe l'envisage est donc fidèle à son principe cardinal de plurivocité, puisqu'il s'agit de convoquer une diversité dont on conserve la singularité, qu'on ne déracine pas de son lieu d'origine, c'est-à-dire d'une partie de son sens.

\section{Le cosmopolitisme au fil de l'œuvre staëlien}

Égérie du Groupe de Coppet, Germaine de Staël est l'auteure d'une œuvre qui abolit les frontières génériques, linguistiques, nationales et politiques, le tout au nom d'une philosophie, d'une éthique et d'une esthétique prônant la pluralité, la plurivocité et la spontanéité au nom de la liberté, fil rouge de son œuvre. Dès De la littérature, Germaine de Staël convoque l'élément national pour expliquer la spécificité de chaque littérature, analyse novatrice qui fera des émules ${ }^{20}$. Principalement déployé à partir de l'Allemagne, l'Italie et l'Angleterre, le cosmopolitisme staëlien investit dès lors chaque genre qu'elle mobilise, de l'essai au roman, de Corinne à Dix années d'exil, en passant, bien sûr, par De l'Allemagne.

\section{L'Allemagne}

Les premiers contacts de Germaine de Staël avec l'Allemagne, en 1804, lui permettent de donner à sa pensée - donc à celle du Groupe une orientation nouvelle; jusque-là, l'écrivaine ne connaissait que les idées littéraires émises en France aux XVII ${ }^{\mathrm{e}}$ et XVIII ${ }^{\mathrm{e}}$ siècles. La mise en contact avec l'Allemagne donne une nouvelle impulsion à sa pensée, donc à celle de Coppet, dont sortira une critique, puis une littérature nouvelles, prônant la spontanéité, le goût relatif et la nécessité de penser les littératures nationales en fonction des usages de la nation qui les voit naître, sans les comparer à l'aune d'un patrimoine garant d'un bon goût absolu et universel que l'écrivaine tient pour une

19. Stephen Bann, «Théorie et pratique de la traduction au sein du Groupe de Coppet », dans Simone Balayé et Jean-Daniel Candaux (dir.), Le Groupe de Coppet, op. cit., p. 217-233; ici p. 221.

20. Axel Blaeschke, «Littérature et liberté: l'engagement selon Madame de Staël et Benjamin Constant», dans Simone Balayé et Jean-Daniel Candaux (dir.), Le Groupe de Coppet, op. cit., p. 417-447; ici p. 421. 
chimère, considérant qu'aucune nation ne devrait imiter la littérature française «qui, par ses beautés mêmes, ne convient qu’aux Français ${ }^{21}$ ».

Ce faisant, Germaine de Staël, et le Groupe à sa suite, renient la doctrine de l'imitation et les genres établis au XVII ${ }^{\mathrm{e}}$ siècle, et donnent à l'écrivain un rôle politique, symbolique et sociall22; ils s'opposent donc fermement au système critique classique que cherche à réimposer le régime impérial, qui, suivant La Harpe, tient le goût français pour le goût absolu, universel ${ }^{23}$, le modèle auquel les autres nations devraient se plier. À cette imitation imposée, à l'émulation factice prônée par le régime, Coppet oppose l'invention, l'inspiration («dans ce qu'ils [les auteurs] ont de plus intime au fond de l'âme $\left.{ }^{24} »\right)$ et le pouvoir de l'imagination à subvertir ce qui est au profit de ce qui devrait être, selon le principe de la perfectibilité. Le Groupe rejette également la vision du régime selon laquelle l'écrivain se doit d'être apolitique; à la tentation de monopolisation des productions intellectuelles et artistiques qui habite très tôt le pouvoir consulaire, puis impérial, le Groupe oppose l'universel et la liberté; face au monopole du national, et aux carcans que cela implique, il promeut le cosmopolite et l'universel, et la liberté qu'ils promettent - « [c]ar nous n’en sommes pas, j’imagine, à vouloir élever autour de la France littéraire la grande muraille de la Chine, pour empêcher les idées du dehors d'y pénétrer ${ }^{25}$ ", écrit Staël dans un passage de De l'Allemagne qui devait être censuré.

Aussi comprend-on aisément pourquoi on a longtemps opposé les concepts de nationalisme et de patriotisme, qui sont le fait de peuples fermés sur eux-mêmes, à celui de cosmopolitisme: De l'Allemagne, pour Savary, n'est pas un ouvrage français, du fait même qu'il ose, dans son appel à renouveler le littéraire par-delà les frontières politiques, convoquer d'autres littératures nationales à l'appui de son propos. Pour le régime impérial, De l'Allemagne est un ouvrage antinational par son refus des valeurs patriotiques qui fondent la politique de conquête impériale; refuser des valeurs patriotiques présentées comme com-

21. Germaine de Staël, Dix années d'exil, Paris, Payot et Rivages, coll. «Rivages poche », 2012, p. 233.

22. Voir Simone Balayé, "Le Groupe de Coppet», art. cit., p. $3^{8 .}$

23. Voir id.

24. Germaine de Staël, Dix années d'exil, op. cit., p. 233.

25. Germaine de Staël, passage censuré de De l'Allemagne, établissement du texte, notes et avant-propos de Simone Balayé, Paris, Garnier-Flammarion, 1968, t. I, p. 47. 
munes, c'est pour l'Empereur faire preuve d'esprit de parti ${ }^{26}$, c'est-àdire récuser les positions adverses à son parti pour la seule raison qu'elles émanent du camp adverse. Or, c'est bien cette rhétorique manichéenne qui s'impose durablement dans la postérité, pour qui cosmopolitisme et patriotisme feront longtemps figure de termes antagonistes.

\section{L'Italie}

Ce soupçon d'antinationalisme, qui devait entacher durablement la postérité staëlienne, s’applique également à Corinne; publié en 1807, ce roman a pour titre complet Corinne ou l'Italie, titre révélateur du projet staëlien, puisque le « ou» permet d'unir l'individu à la culture, le particulier à l'universel plutôt que de les dissocier ${ }^{27}$. Aussitôt paru, le roman s'attire les foudres de l'empereur, et ce pour un motif simple; dans ce texte, il n'est pas une fois question des troupes françaises qui occupent alors une partie du pays. Il s'agit bien plutôt d'un roman européen, où diverses nations coexistent et dialoguent sans que l'une ne s'impose face aux autres comme un modèle à suivre. Dans cette perspective, chaque personnage constitue l'allégorie d'une nation, et plus précisément d'une caractéristique nationale, occasion pour l'écrivaine de faire le tableau des mœurs et de la culture qu'il représente; le comte d'Erfeuil, seul Français présent, est ainsi pointé du doigt pour sa tendance à la dérision et sa frivolité, mais aussi pour sa volonté de tout inféoder au goût français, modelé sur le siècle de Louis XIV, indépassable à ses yeux - comme à ceux du régime impérial. Si lord Nelvil représente la mélancolie et le respect des convenances propres à l'Angleterre, Corinne, allégorie de l'Italie, incarne son enthousiasme et son amour de l'art et du génie, que la future nation italienne ne rechigne jamais à célébrer, même lorsqu'il s'incarne en une femme; ses origines anglaises permettent quant à elles au roman de conserver son dynamisme dialogique. Ce passage situé lors du triomphe de Corinne le montre assez: «L'émotion était générale; mais lord Nelvil

26. Voir Florence Lotterie, «Madame de Staël et l'esprit de Coppet: une littérature d'“opposition"? ", dans Jean-Claude Bonnet (dir.), L'empire des Muses. Napoléon, les arts et les lettres, Paris, Belin, 2004, p. 133-149; ici p. 138.

27. Voir Brigitte Hermann, "Corinne et la pratique du roman européen », dans Kurt Kloocke (dir.), Le Groupe de Coppet et l'Europe, op. cit., p. 229-237; ici p. 234. 
ne la partageait point encore; et bien qu'il se fût déjà dit qu'il fallait mettre à part, pour juger tout cela, la réserve de l'Angleterre, et les plaisanteries françaises, il ne se livrait point à cette fête, lorsqu'enfin il aperçut Corinne ${ }^{28} »$. Si Nelvil représente la réserve propre à l'Angleterre, Erfeuil figure les «plaisanteries françaises ${ }^{29}$ »; Corinne, quant à elle, suscite (et incarne) l'enthousiasme propre aux Italiens.

Corinne constitue à ce titre le roman le plus caractéristique du cosmopolitisme staëlien: par son processus de création, d'abord, puisqu'il est né à la suite d'un voyage en Italie et prend donc sa source, comme De l'Allemagne, dans l'observation directe de mœurs et de cultures étrangères; par son exaltation du pluriel et du cosmopolite ensuite, puisque chaque nation voit ses spécificités représentées, unies dans leur indépendance et dans leur liberté; par sa célébration de l'universalité du génie, enfin, puisque celui-ci ne connaît réellement aucune frontière - qu'elle soit linguistique, politique ou genrée. C'est là, en somme, ce que Germaine de Staël, à l'image de Corinne, «femme illustrée seulement par les dons du génie ${ }^{30}$ », s'est appliquée à montrer toute sa vie durant.

\section{L'Angleterre}

On trouve également et surtout, au fondement du cosmopolitisme de Germaine de Staël, son amour de l'Angleterre, qu'elle pose comme le seul pays en Europe à avoir fait de la liberté le socle de la vie sociale et politique $^{31}$. Représentative des modalités du cosmopolitisme staëlien, la connaissance qu’a Germaine de Staël de l’Angleterre se développe à travers ses voyages, mais aussi au gré de ses lectures et grâce à son cercle d'amis ${ }^{32}$. L'histoire de l'Angleterre prouve que la liberté, cause pour laquelle elle n'a cessé de lutter, n'est pas une chimère: puisque la liberté existe en Angleterre, elle n’est pas un idéal inaccessible pour

28. Germaine de Staël, Corinne ou l'Italie, établissement du texte, notes et avantpropos de Simone Balayé, Paris, Champion, 2000, p. 25.

29. Id.

30. Ibid., p. 26.

31. Victor de Pange, «Le rêve anglais de Madame de Staël», dans Madame de Staël et l'Europe. Colloque de Coppet (18-24 juillet 1966) organisé pour la célébration du deuxième centenaire de la naissance de Madame de Staël (1766-1966), Paris, Klincksieck, 1970, p. 173-192; ici p. 173.

32. Voir id. 
la France ${ }^{33}$; de façon éloquente, Mme Necker de Saussure écrira d'ailleurs que «[1]'Angleterre n'est à ses yeux que la France future ${ }^{34}$ ».

Chez l'écrivaine, la réflexion sur la littérature, la société, la philosophie anglaises, sert dès lors systématiquement un propos politique - au prix, souvent, d'un langage idéalisant ${ }^{35}$ : l'objectif est bien d'ériger un «idéal pour combattre ${ }^{36}$ » le modèle impérial. De l'Allemagne appelle ainsi aux armes contre ce nouvel Attila qu'est Napoléon ${ }^{37}$ par le biais de l'exemple britannique, distillé au gré d'allusions plus ou moins explicites. Car admirer ce «grand peuple libre », c'est dénoncer le fait que les Français ne le sont plus; professer son goût pour le sens civique anglais, ces institutions politiques où législatif et exécutif s'équilibrent mutuellement, exprimer sa satisfaction à voir le roi régner sans gouverner ${ }^{38}$, c'est faire, en creux, le portrait - sinon le procès - de la France impériale, et lui indiquer le modèle à suivre.

\section{La traduction}

Modalité-clé du cosmopolitisme de Coppet, sa conception de la traduction occupe également une place de choix dans la pensée staëlienne. Publié dans un journal italien en 1816, l'article « De l'esprit des traductions » est l'analyse la plus aboutie de la théorie de la traduction selon Germaine de Staël ${ }^{39}$. Traitant de la littérature italienne et des problèmes inhérents à la culture transalpine, l'article s'ouvre sur une défense du principe de la traduction: «Il n'y a pas de plus éminent service à rendre à la littérature, que de transporter d'une langue à l'autre les chefs-d'œuvre de l'esprit humain ${ }^{40}$ ", écrit l'auteure. Du fait

33. Voir Norman King, "Madame de Staël et la chute de Napoléon », dans Madame de Staël et l'Europe, op. cit., p. 63-79; ici p. 76 .

34. Albertine Necker de Saussure, "Notice sur le caractère et les écrits de Madame de Staël », dans (Euvres posthumes de Madame la Baronne de Staël-Holstein, Paris, Firmin Didot, 1844, p. 2-54; ici p. 29.

35. Voir Simone Balayé, "Madame de Staël et l'Europe napoléonienne », dans Kurt Kloocke (dir.), Le Groupe de Coppet et l'Europe, op. cit., p. 25-37; ici p. 30.

36. Id.

37. Voir Victor de Pange, «Le rêve anglais de Madame de Staël », art. cit., p. 186.

38. Voir id.

39. Voir Stephen Bann, «Théorie et pratique de la traduction au sein du Groupe de Coppet», art. cit., p. 223.

40. Germaine de Staël, «De l'esprit des traductions », dans (Euvres complètes de Madame la Baronne de Staël-Holstein, Paris, Treuttel et Würtz, 1821, t. XVII, p. $3^{87-}$ 399 ; ici p. $3^{87}$. 
du nombre réduit de chefs-d'œuvre, les nations ne peuvent se borner à leurs propres chefs-d'œuvre littéraires; dès lors, la traduction, modalité emblématique du cosmopolitisme, est nécessaire aux nations: «de tous les genres de commerce », elle constitue «celui dont les avantages sont les plus certains ${ }^{41} »-$ ce qui s'oppose directement au principe d'uniformité forcée du régime impérial et aux productions banales et médiocres qui en ont résulté. Même dans l'hypothèse où l'on connaîtrait parfaitement toutes les langues étrangères, la traduction, pratique résolument cosmopolite, resterait essentielle en tant que vecteur d'un style, d'un plaisir différents, mais aussi comme un jalon essentiel du renouvellement de la littérature nationale: «Ces beautés naturalisées donnent au style national des tournures nouvelles, et des expressions plus originales. Les traductions des poètes étrangers peuvent, plus efficacement que tout autre moyen, préserver la littérature d'un pays de ces tournures banales qui sont les signes les plus certains de sa décadence $^{42} \gg$.

C'est bien ce que Germaine de Staël a fait dans De l'Allemagne pour la prose et le théâtre allemands, et dans d'autres ouvrages pour la poésie lyrique. Or l'ouverture aux langues étrangères, capitale aux yeux de l'écrivaine, a été trop souvent négligée en France: «il ne faut pas, comme les François, donner sa propre couleur à tout ce qu'on traduit; quand même on devroit par là changer en or tout ce que l'on touche, il n'en résulteroit pas moins que l'on ne pourroit pas s'en nourrir; on n'y trouveroit pas des aliments nouveaux pour sa pensée ${ }^{43}$ ». Tout Coppet est dans cette phrase : loin de la couleur uniforme, mortifère à la pensée, à l'élargissement de la perspective et à l'enrichissement des idées, il faut chercher et préserver, dans ses traductions également, la couleur propre à chaque langue, chaque terroir, chaque pays, puisque, en traduisant une œuvre, on transpose également une culture, des mœurs et une histoire spécifiques. L'avenir de la littérature, de la pensée, réside tout entier dans l'innovation, la création, le renouvellement, domaines dans lesquels la traduction est appelée à jouer un rôle essentiel; il faut dès lors entendre la traduction selon Staël comme un projet à la fois herméneutique et philanthropique.

41. Id.

42. Ibid., p. $3^{89}$.

43. Id. 
Si l'on doit circonscrire à partir de l'œuvre staëlien une spécificité du cosmopolitisme féminin, il faut peut-être la chercher dans ce recours conjoint et complémentaire de l'écrivaine aux essais et articles, qui permettent la théorisation d'un discours d'ouverture à l'autre (nation, langue, culture), pluriel dans son essence même, et aux romans, qui illustrent de façon plus implicite le bienfondé de la pensée cosmopolite, sa pertinence et son rôle clé dans la théorie de la perfectibilité. Alliées à des fins de démonstration, théorie et fiction acquièrent de la sorte une plus grande efficacité, proposant une réflexion qui recouvre de nombreux domaines, et touche différents publics en mobilisant pour convaincre plusieurs procédés, qui jouent tantôt davantage sur l'imagination et la sensibilité, tantôt sur la rhétorique et la logique de la démonstration.

Cette habileté, déployée dans tout le corpus staëlien, laisse entrevoir la cohésion et la cohérence des principes autour desquels l'écrivaine a édifié son œuvre: liberté et aspiration à l'universel se trouvent ainsi conjuguées au service du bien commun, du progrès intellectuel, de l'édification d'un avenir commun, où l'individualisme et l'uniformisme du régime impérial ne feraient plus d'émules. Cosmopolitisme dont le catalyseur aura été l'exil - d'où ses modalités particulières: le réseau de Germaine de Staël s'est concrétisé au cours de voyages, qui, bien que contraints, ont contribué à l'agrandir considérablement, au point de faire de l'écrivaine une citoyenne du monde, figure fédératrice pour les notables et intellectuels de toute l'Europe. Plus qu'une théorie, le cosmopolitisme staëlien est donc une expérience pleinement incarnée, qui prend toute son ampleur au gré des exils de l'écrivaine et influence, ce faisant, l'ensemble du Groupe et l'Europe libérale; en cela, il déploie dans toutes ses potentialités le cosmopolitisme de Coppet, dont il constitue sans doute la forme la plus aboutie.

\section{Conclusion}

\section{Ouverture}

Foyer intellectuel cosmopolite et libéral, Coppet fut donc, on l'aura saisi, un creuset propice à la naissance du romantisme, à la dynamique qui lui est propre et qui consiste en un mouvement perpétuel de création, de réinvestissement, de réinterprétation, d'ouverture à l'autre, de brouillage des frontières dans le sens d'un renouvellement, d'un rajeu- 
nissement de la littérature. Le mot d'ordre du Groupe, mais aussi du romantisme, sera en effet l'ouverture à la diversité par-delà les frontières - esthétiques, linguistiques, politiques et nationales; en cela, le Groupe jette les jalons philosophiques et éthiques de l'Europe moderne. Pour ses membres, l'émergence des nations est synonyme de liberté; à l'échelle d'une histoire allant vers la liberté universelle, la fondation des nations est selon la conviction cosmopolite du Groupe un moment clé de l'émancipation de l'humanitét ${ }^{4}$, dans une perspective non plus seulement européenne, mais bien universelle. En cela, le cosmopolitisme de Coppet est résolument novateur et tourné vers l'avenir : il contribue à redéfinir, en y ajoutant un pan politique ${ }^{45}$, les contours d'une République des Lettres sans frontières, dont on pourrait être membre à la seule condition d'avoir du talent, mais également d'une littérature désormais tournée vers l'autre et l'ailleurs comme autant d'«aliments nouveaux pour sa pensée ».

\section{Synthèse et unité}

S'il doit beaucoup à cette volonté d'ouverture, le caractère novateur de la pensée cosmopolite coppétienne et staëlienne réside également dans son esprit de synthèse. On trouve en effet au sein de l'œuvre staëlien, ainsi que dans les autres productions du Groupe, cette volonté inédite de synthétiser les aspects les plus progressistes du XVIII ${ }^{\mathrm{e}}$ siècle - l'esprit de conversation, les acquis sociaux de la Révolution, la volonté de diffuser les lumières - et les perspectives les plus prometteuses du siècle qui s'ouvre: la pluralité, la diversité, le goût du particulier, du relatif, l'éveil de nations unies dans l'indépendance et la liberté ${ }^{46}$ ou encore la circulation des savoirs.

Car le siècle nouveau semble rendre possible l'émergence d'une Europe selon le vœu du Groupe: éclairée, unie, riche et forte de sa plurivocité même, où les idées et les hommes circuleraient librement; où Coppet, pôle de la pensée, de l'écriture et de l'action, serait le

44. Voir Umberto Marcelli, «Il Gruppo di Coppet e il concetto di nazionalità », dans Simone Balayé et Jean-Daniel Candaux (dir.), Le Groupe de Coppet, op. cit., p. 411.

45. Voir Roland Mortier, «Les États Généraux de l'opinion européenne», art. cit., p. 23.

46. Voir Simone Balayé, «Madame de Staël et l'Europe napoléonienne», art. cit., p. 27. 
carrefour du commerce des idées; où la liberté d'expression (notamment pour l'écrivain) serait de rigueur, et où la discussion serait vivement encouragée en tant que garante de l'esprit démocratique. Lesprit de synthèse propre au cosmopolitisme de Coppet est donc un esprit de communion, d'unité entre les nations et les individus: c'est en effet non vers l'uniformité, mais vers l'unité que tend le Groupe, comme le montre Bonstetten lorsqu'il exprime sa conviction que l'Europe et le monde «ne formeront plus qu'un seul peuple, marchant de concert vers le soleil des naissantes lumières ${ }^{47}$ », avec « une même âme ${ }^{48}$ ».

\section{Le cosmopolitisme de Coppet, idéal total}

Par son esprit cosmopolite, synonyme d'ouverture et d'unité, Coppet est aussi - et peut-être surtout - le creuset d'une perspective nouvelle sur les choses et les peuples, consistant à valoriser la spécificité, et non plus à en évaluer l'écart face à un quelconque absolu du goût. Cette capacité d'un regard autre, donc d'une possible subversion, tient pour partie à la position frontalière de Coppet: de même que la subversion de Voltaire devait beaucoup à la situation de Ferney, c'est grâce à sa position décentrée que le château de Germaine de Staël, à la fois proche et éloigné de la France, peut porter un regard sur sa situation politique à la fois depuis le dedans et le dehors ${ }^{49}$. Le cosmopolitisme coppétien a en effet cela de singulier qu'il résulte de la reconstitution à l'échelle européenne, dans un lieu frontalier et décentré, de l'espace public dont l'écrivaine s'est vue privée en France.

Placé sous le signe de l'universel, le cosmopolitisme de Coppet, exploration internationale, pluridisciplinaire et multigenre de la pluralité, relève de l'idéal total, du projet intellectuel, esthétique et civilisationnel, car l'uniformisme que combat le Groupe touche à des considérations à la fois linguistiques, idéologiques et politiques. Il s’agit ainsi de défendre une éthique nouvelle qui se fonderait sur un idéalisme transcendantal, sur une morale du sacrifice, de la générosité et du respect de l'homme ${ }^{50}$. Cela, Pierre-Simon Ballanche l'a bien saisi, qui écrit:

47. Charles Victor de Bonstetten, Études de l'homme, Genève/Paris, J. J. Paschoud, 1821 , t. I, p. 278.

48. Ibid., p. 288.

49. Voir Roland Mortier, «Les États Généraux de l'opinion européenne », art. cit., p. 19.

5o. Voir id. 
Coppet [...] serait le berceau de la société nouvelle. Cette frontière des idées allemandes et des idées françaises, des sentiments allemands et des sentiments français serait aussi la frontière des idées anciennes et des idées nouvelles, des sentiments anciens et des sentiments nouveaux... C'est là aussi que l'on trouvera la fin du règne classique et le commencement du règne romantique. Le personnage de $\mathrm{M}^{\text {me }}$ de Staël aura alors toute son importance historique ${ }^{51}$.

Coppet est donc, par son cosmopolitisme, un lieu de recommencement, de renouvellement civilisationnel, le creuset du romantisme, le berceau des idées nouvelles. Pour ce Groupe d'un genre nouveau, la littérature constitue décidément «le territoire de l'universel humain face à l'égoïsme érigé en système par l'Empire ${ }^{52}$ ». En effet, pour le Groupe, l'écrivain est le porte-parole de l'universel, d'une Europe qu'il s'agit de défendre contre «l'Europe France ${ }^{53}$ ", c'est-à-dire contre la prétention de l'Empire à définir et à incarner le monopole du rayonnement de la France sur la scène européenne ${ }^{54}$. Pour le Groupe, la littérature et la culture, par-delà leurs spécificités nationales, rassemblent les hommes et contribuent au progrès d'une civilisation commune, en vertu du principe cardinal de perfectibilités5. D’un cosmopolitisme centrifuge, où l'unification de l'Europe s'effectuerait par la francisation, on passe ainsi à un cosmopolitisme pluridirectionnel, à une conception polyphonique de la culture où l'unité serait le fruit de l'union des particularismes nationaux, et non de leur effacement $^{56}$. L'Europe selon Coppet est donc une Europe des nationalités, mais plus encore des talents et des mérites ${ }^{57}$.

51. "Lettre de Pierre-Simon Ballanche à Juliette Récamier du 27 février 1819", dans Édouard Herriot, Madame Récamier et ses amis, Paris, Plon, 1913, t. II, p. 65.

52. Florence Lotterie, «Madame de Staël et l'esprit de Coppet: une littérature d'“opposition"? ", art. cit., p. 135.

53. Germaine de Staël, Dix années d'exil, op. cit., p. 133.

54. Voir Florence Lotterie, «Madame de Staël et l'esprit de Coppet: une littérature d'“opposition"?", art. cit., p. 139.

55. Voir ibid., p. 138.

56. Voir Roland Mortier, «Les États Généraux de l'opinion européenne», art. cit., p. 19.

57. Voir Georges Andrey, «Madame de Staël et l'émigration : essai d'inventaire des problèmes », dans Étienne Hofmann et Anne-Lise Delacrétaz (dir.), Le Groupe de Coppet et la Révolution française. Actes du quatrième Colloque de Coppet, 20-23 juillet 1988, Lausanne/Paris, Institut Benjamin Constant/Touzot, 1988, p. 15-34; ici p. 32 . 
Ainsi, ce que vise le cosmopolitisme coppétien, c'est peut-être simplement la possibilité que la différence serve de socle à un projet commun aux hommes qui pensent à l'échelle européenne - le culte de la vérité et de la beauté -, que les spécificités nationales servent à surmonter les frontières comme autant d'obstacles à une communion des nations et des individus. Quelles que soient les circonstances, et jusque dans l'exil, il serait donc légitime, selon le Groupe et son inspiratrice, d'espérer et de penser, en vertu de la perfectibilité, que «[1]e mal des lumières ne peut se corriger qu'en acquérant plus de lumières encore $^{58} »$. Leçon pertinente, alors et de tout temps.

58. Germaine de Staël, De la littérature considérée dans ses rapports avec les institutions sociales, établissement du texte, notes et avant-propos d'Axel Blaeschke, Paris, Garnier, coll. «Classiques Jaunes», 2014, p. 328. 